\title{
Scissors modes of two-component degenerate gases: Bose-Bose and Bose-Fermi mixtures
}

\author{
M. Rodríguez ${ }^{1}$, P. Pedri ${ }^{2,3}$, P. Törmä ${ }^{4}$ and L. Santos ${ }^{2}$ \\ (1) Laboratory of Computational Engineering, P.O. Box 9203, \\ FIN-02015 Helsinki University of Technology, Finland
}

(2) Institut für Theoretische Physik, Universität Hannover, D-30167 Hannover,Germany

(3) Dipartimento di Fisica, Università di Trento and BEC-INFM, I-38050 Povo, Italy and

(4) Department of Physics, University of Jyväskylä, P.O.Box 35, FIN-40014 Jyväskylä, Finland

\begin{abstract}
We investigate the scissors modes in binary mixtures of degenerate dilute quantum gases, for both Bose-Bose and Bose-Fermi mixtures. For the latter we consider both the superfluid and normal hydrodynamic and collisionless regimes. We analyze the dependence of the frequencies of the scissors modes and their character as a function of the Bose-Fermi coupling and the trap geometry. We show that the scissors mode can reveal a clear trace of the hydrodynamic behavior of the Fermi gas.
\end{abstract}

\section{INTRODUCTION}

In recent years, the improvement of the trapping techniques has allowed for the creation of multi-component Bose-Einstein condensates (BECs), formed by atoms in different internal (electronic) states [1, 2]. The multicomponent BEC, far from being a trivial extension of the single-component one, presents novel and fundamentally different scenarios for its ground-state wavefunction 3, 4, 5] and excitations 6, 7, 8. In particular, it has been experimentally observed that the BEC can reach an equilibrium state characterized by the phase separation of the species in different domains 2].

During the last few years, the mixtures of fermions and bosons, from now on called Fermi-Bose (FB) mixtures, have also attracted a growing attention. Although the initial interest has been mostly motivated by the possibility to achieve sympathetic cooling towards the Bardeen-Cooper-Schriffer (BCS) transition [9, 10], recently, the rich physics of FB mixtures has become itself one of the central topics of the physics of ultracold gases. Various properties of the FB mixtures have been analyzed, including the phase separation between bosons and fermions 11, 12, 13 , the existence of novel types of collective modes [12, 13, 14, 15], the appearance of effective Fermi-Fermi interactions mediated by the bosons [12, 13, 16, 17], the collapse of the Fermi cloud in the presence of attractive interactions between bosons and fermions 18], the physics of 1D FB mixtures 19] or FB mixtures in optical lattices [20].

The analysis of collective excitations is an excellent tool to analyze the effects of interactions in ultracold dilute gases. Zero-temperature excitation frequencies have been extensively studied in the case of Bose-Einstein condensates (BECs) in dilute alkali gases up to a high degree of experimental accuracy [21, 22]. These experimental results are in excellent agreement with the theoretical predictions obtained from first principles [23, 24, 25].

Among the different collective modes the so-called scissors mode has attracted considerable attention. This mode is well known in nuclear physics 26], where it corresponds to the out-of-phase rotation of the neutron and proton clouds. In the context of ultracold atomic gases, the scissors mode is achieved after a sudden tilt of the trap, and it consists in the harmonic motion of the atomic cloud around the new trap axis. This particular collective excitation, predicted in Ref. 27], has been successfully observed experimentally [28, 29].

The present paper is devoted to the analysis of the scissors modes in mixtures of degenerate atomic gases, both for the case of a multicomponent BEC, and for a FB mixture. In the latter case, we analyze both when the Fermi gas is in hydrodynamic and in collisionless regime. We discuss in particular the variation of the frequencies of the scissors modes (as well as their associated geometry) as a function of the Bose-Bose (or Bose-Fermi) coupling constant.

The paper is organized as follows. In Sec. II we analyze the case of a Bose-Bose mixture. Sec. III is devoted to the analysis of the scissors modes in FB mixtures, first for the case of a hydrodynamic Fermi gas, and later when the Fermi gas is in the normal phase. We finalize in Sec. IV with our conclusions, and the discussion of the results. In App. A we have included some analytical results, which have been employed in different calculations throughout the paper.

\section{BINARY BOSE-EINSTEIN CONDENSATE}

In this section we consider the case of a BEC formed by two different components $\{1,2\}$, confined in a harmonic external potential

$$
V_{i}(\mathbf{r})=\frac{m_{i}}{2}\left[\omega_{x ; i}^{2} x^{2}+\omega_{y ; i}^{2} y^{2}+\omega_{z ; i}^{2} z^{2}\right]
$$

where $i=1,2$. We are interested in the scissors modes of this particular system after a sudden tilt of the trap axis. We assume that the chemical potential is larger than the energy separation between the trap levels (Thomas-Fermi regime). Therefore the effects of the quantum pressure are negligible, and the system can be analyzed using the 
hydrodynamic equations for superfluids

$$
\begin{aligned}
& \frac{\partial n_{1}}{\partial t}+\nabla \cdot\left(n_{1} \mathbf{v}_{1}\right)=0 \\
& \frac{\partial n_{2}}{\partial t}+\nabla \cdot\left(n_{2} \mathbf{v}_{2}\right)=0 \\
& m_{1} \frac{\partial \mathbf{v}_{1}}{\partial t}+\nabla\left(\frac{m_{1}}{2} v_{1}^{2}+V_{1}+g_{11} n_{1}+g_{12} n_{2}\right)=0 \\
& m_{2} \frac{\partial \mathbf{v}_{2}}{\partial t}+\nabla\left(\frac{m_{2}}{2} v_{2}^{2}+V_{2}+g_{12} n_{1}+g_{22} n_{2}\right)=0
\end{aligned}
$$

where $n_{i}$ is the density, $\mathbf{v}_{i}$ the velocity field, $m_{i}$ the mass, and $V_{i}$ the external potential, associated with the $i$-th component. The coefficient $g_{i j}=2 \pi \hbar^{2} a_{i j} / \tilde{m}_{i j}$ is the coupling constant associated with the interatomic interactions between the components $i$ and $j$, where $\tilde{m}_{i j}$ and $a_{i j}$, are the corresponding reduced mass and scattering length, respectively.

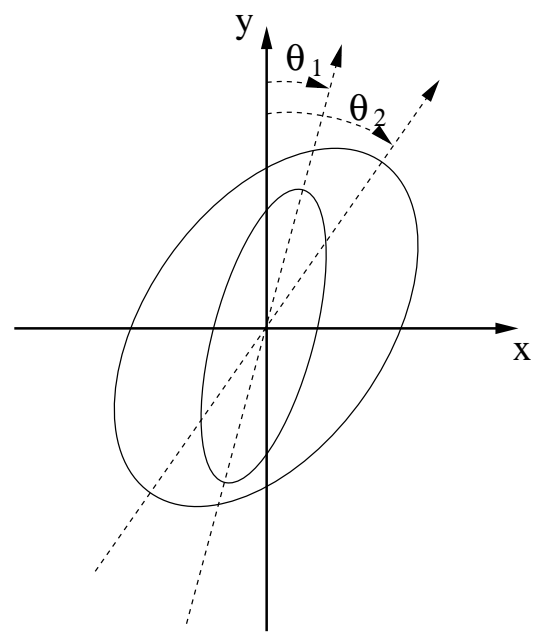

Figure 1: Schematic description of the two angles of rotation involved in the scissors modes.

Neglecting the quantum pressure term in a single component BEC demands that $N a / l_{h o} \gg 1$, where $N$ is the total number of particles and $l_{h o}=\sqrt{\hbar / m \bar{\omega}}$, where $\bar{\omega}^{3}=\omega_{x} \omega_{y} \omega_{z}$. This approximation breaks down at the border of the Thomas-Fermi density profile, where the density is small. In the case of a two-component BEC, one must additionally require that the two species are not strongly phase separated. Otherwise, at the domain wall, the kinetic energy dominates the mean-field potential, and therefore the quantum pressure is certainly not negligible.

Imposing a stationary solution without flow $(\mathbf{v}=\mathbf{0})$, we obtain

$$
\begin{aligned}
& V_{1}(\mathbf{r})+g_{11} n_{1 g}(\mathbf{r})+g_{12} n_{2 g}(\mathbf{r})=\mu_{1} \\
& V_{2}(\mathbf{r})+g_{12} n_{1 g}(\mathbf{r})+g_{22} n_{2 g}(\mathbf{r})=\mu_{2},
\end{aligned}
$$

from where we can easily calculate the ground-state density profiles $n_{i g}(\mathbf{r})$.
Since we are interested in the oscillations of the cloud in the $x-y$ plane after a tilting from the equilibrium solution, it is convenient to adopt the coordinates $\left.x_{i}^{\prime}=x \cos \left(\theta_{i}(t)\right)-y \sin \left(\theta_{i}(t)\right)\right)$ and $y_{i}^{\prime}=x \sin \left(\theta_{i}(t)\right)-$ $\left.y \cos \left(\theta_{i}(t)\right)\right)$. We consider small angles $\theta_{i}$, and consequently we can perform the Ansatz $n_{i}(t, x, y, z)=$ $n_{i g}\left(x_{i}^{\prime}, y_{i}^{\prime}, z\right) \simeq n_{g}(x, y, z)+\theta_{i}\left(y \partial_{x}-x \partial_{y}\right) n_{g x}(x, y, z)$. Note that the time dependence is entirely contained in the parameters $\theta_{1}$ and $\theta_{2}$ (see Fig. 1).

After multiplying the continuity equations by $x y$ and integrating, we obtain the equation for the asymmetry of the cloud of the $i$-th component

$$
\frac{d}{d t}\langle x y\rangle_{i}=\left\langle x v_{y}+y v_{x}\right\rangle_{i} .
$$

Note that the asymmetry parameter is related to the angular displacement $\theta_{i}$ in the form $\langle x y\rangle_{i}=\left\langle y^{2}-x^{2}\right\rangle_{g} \theta_{i}$, where $\langle\ldots\rangle_{g}$ denotes the expected value in the equilibrium state. Neglecting terms of second order in the angular displacements, we obtain

$$
\frac{d}{d t}\left\langle x v_{y}+y v_{x}\right\rangle=\frac{1}{N} \int(y, x, 0) \cdot\left[\frac{\partial \mathbf{v}}{\partial t}\right] n d^{3} r .
$$

Substituting into Eq. (9) the corresponding Euler equation for the $i$-th components, and combining with Eqs. (8), we obtain, in the linear regime

$$
\begin{aligned}
& B_{11} \ddot{\theta}_{1}=A_{11} \theta_{1}+A_{12} \theta_{2} \\
& B_{22} \ddot{\theta}_{2}=A_{21} \theta_{1}+A_{22} \theta_{2},
\end{aligned}
$$

where the coefficients $A_{i j}$ and $B_{i}$ can be found in App. A By imposing a solution of the form $\exp \{-i \omega t\}$, we find the frequencies of the different scissors modes

$$
\omega_{ \pm}=\frac{A_{11} B_{2}+A_{22} B_{1} \pm \sqrt{\left(A_{11} B_{2}+A_{22} B_{1}\right)^{2}-\operatorname{det}[\mathbf{A}]}}{B_{1} B_{2}},
$$

where we have introduced the matrix $\mathbf{A}=\left\{A_{i j}\right\}$. The corresponding eigenvectors $\left\{\theta_{1}^{ \pm}, \theta_{2}^{ \pm}\right\}$provide information about the nature of the modes. The two components oscillate in-phase when $\theta_{1} \theta_{2}>0$, and in counter-phase, when $\theta_{1} \theta_{2}<0$.

In Fig. 2 we depict our results for a particular set of parameters (see figure caption). Both in-phase and outof-phase modes depend on the particular value of $g_{12}$. Notice, however, that the in-phase mode is less affected by the change of $g_{12}$, since the interaction energy between both gases is just slightly modified during the motion. In particular, if the trapping frequencies for both components are the same, the in-phase mode remains independent of the interaction coupling constant $g_{12}$. The counter-phase mode is in any case strongly affected.

The in-phase mode is always the lowest energy state for $g_{12}<0$, since it maximizes the overlapping. For $g_{12}>0$, the out-of-phase mode becomes the state with lowest energy, as long as the two components present a maximum in the trap center, since in that case the out-ofphase mode minimizes the overlapping. For a given value 
$g_{12}=g_{12}^{c r}$, the density profile of the component with lowest central density (say the component 2) acquires zero curvature at the trap center, therefore, for $g_{12}>g_{12}^{c r}$, one of the components presents a minimum at the trap center, and the in-phase mode becomes once more the lowest energy state. For $\omega_{x, y, z ; 1}=\omega_{x, y, z ; 2}, g_{12}^{c r}=g_{11}$, whereas from the Thomas-Fermi solutions (6) and (7), one can easily obtain that in general, if $\beta=\omega_{x, y, z ; 1} / \omega_{x, y, z ; 2}$, $g_{12}^{c r} / g_{11}=\beta^{2}\left(m_{1} / m_{2}\right)$. This dependence of the modes has been confirmed by our numerical simulations (see Fig. 2).

\section{BOSE-FERMI MIXTURE}

In this section we analyze the scissors modes for FB mixtures. Two different regimes are considered: (i) The case in which the fermions are in the hydrodynamic regime, and (ii) the case in which the fermions are in the normal phase in the collisionless regime. Concerning the case (i), we would like to note that the scissors modes for a FB mixture in the normal phase in the hydrodynamic regime, coincide with those modes of the superfluid hydrodynamics. In Sec. IV we comment on this issue in more detail.

\section{A. Hydrodynamic regime}

In a two-component Fermi gas, if the typical collisional time becomes much larger than the trapping period, the Fermi gas is said to enter into the hydrodynamic regime. As for the Bose-Bose mixture, the FB mixture in the hydrodynamic regime can be well described by means of the hydrodynamic equations

$$
\begin{gathered}
\frac{\partial n_{b}}{\partial t}+\nabla \cdot\left(n_{b} \mathbf{v}_{b}\right)=0 \\
\frac{\partial n_{f}}{\partial t}+\nabla \cdot\left(n_{f} \mathbf{v}_{f}\right)=0 \\
\frac{\partial \mathbf{v}_{b}}{\partial t}+\nabla\left(\frac{v_{b}^{2}}{2}+\frac{V_{b}+g_{b} n_{b}+g_{b f} n_{f}}{m_{b}}\right)=0 \\
\frac{\partial \mathbf{v}_{f}}{\partial t}+\nabla\left(\frac{v_{f}^{2}}{2}+\frac{V_{f}+g_{b f} n_{b}}{m_{f}}+\frac{\hbar^{2}}{2 m_{f}^{2}}\left(6 \pi^{2} n_{f}\right)^{2 / 3}\right)= \\
=\mathbf{v}_{f} \wedge\left(\nabla \wedge \mathbf{v}_{f}\right)
\end{gathered}
$$

Note that in our calculations only one set of equations is considered for the Fermi gas. This is justified if the difference between the trapping frequencies and the concentrations of both Fermi components is negligible [30]. Note also that we have employed the chemical potential for a free Fermi gas, $\sim n_{f}^{2 / 3}$, since in the quantum degeneracy regime due to Pauli-blocking (away from Feshbach resonances) the corrections due to Fermi-Fermi interactions are small.

As for the Bose-Bose mixture, we obtain the ground state density profile in the Thomas-Fermi approximation,
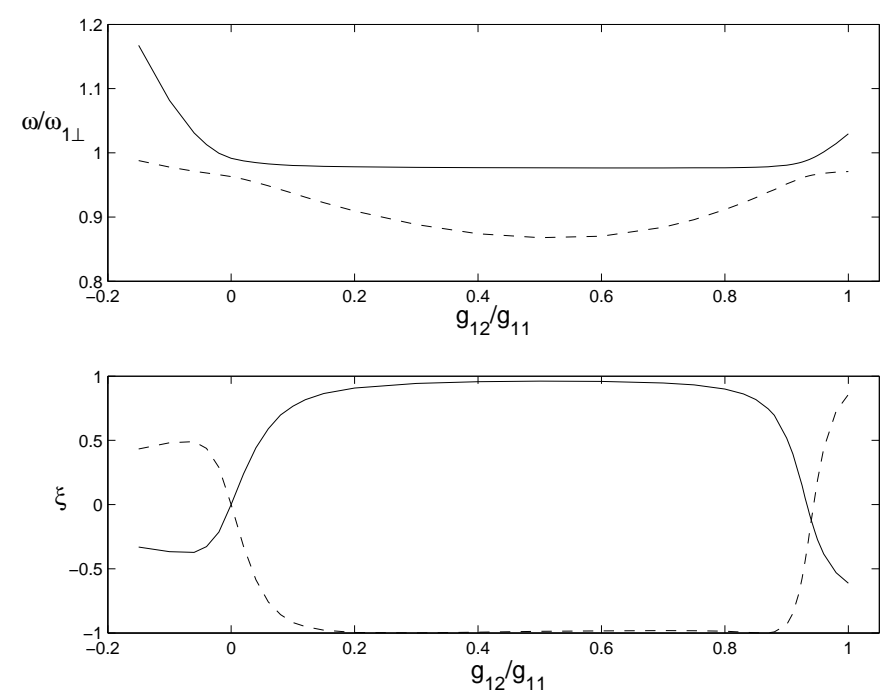

Figure 2: (a) Frequencies of the scissors modes for a binary BEC as a function of the intercomponent coupling constant $g_{12}$ for $N_{1}=N_{2}=10^{6}, \omega_{x, y, z ; 2}=0.97 \omega_{x, y, z ; 1}$, equal masses $m, \omega_{y ; 1}=1.5 \omega_{x ; 1}$, and $\omega_{z ; 1}=2 \omega_{x ; 1}$. The dimensionless interaction energies are $g_{11} / \hbar \omega_{x ; 1} l_{x ; 1}^{3}=1$ and $g_{22}=2 g_{11}$, where $l_{x ; 1}^{2}=\hbar / m \omega_{x ; 1}$. The frequencies are indicated in units of $\omega_{1 \perp}=\left(\omega_{x ; 1}^{2}+\omega_{y ; 1}^{2}\right)^{1 / 2}$. (b) Character of the modes, provided by $\xi=2 \theta_{1} \theta_{2} /\left(\theta_{1}^{2}+\theta_{2}^{2}\right)$, as a function of $g_{12}$. The in-phase (out-of-phase) mode is characterized by $\xi>0(\xi<0)$.
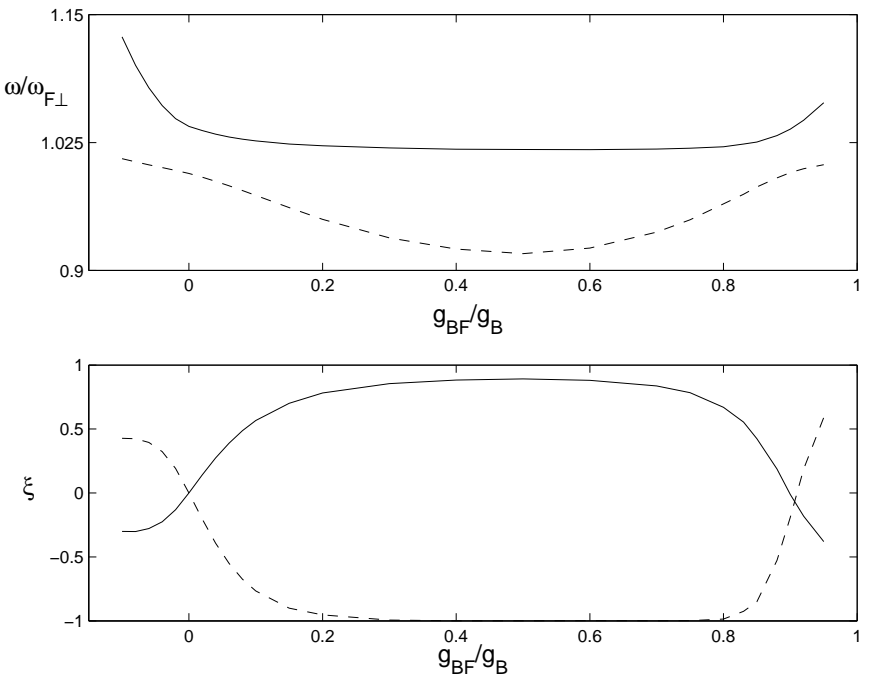

Figure 3: (a) Frequencies of the scissors modes for a FB mixture, where the Fermi gas is in the hydrodynamic regime, in units of $\omega_{F \perp}=\left(\omega_{x ; f}^{2}+\omega_{y ; f}^{2}\right)^{1 / 2}$, as a function of the intercomponent coupling constant $g_{b f}$; (b) Character of the modes, provided by $\xi=2 \theta_{b} \theta_{f} /\left(\theta_{b}^{2}+\theta_{f}^{2}\right)$, as a function of $g_{b f}$. The in-phase (out-of-phase) mode is characterized by $\xi>0$ $(\xi<0)$. In the figures we consider the case $N_{b}=N_{f}=10^{6}$, equal masses $m, \omega_{x, y, z ; b}=1.05 \omega_{x, y, z ; f}, \omega_{x ; f}=0.1 \omega_{z ; f}$, $\omega_{y ; f}=0.995 \omega_{z ; f}$, and $g_{b} / \hbar \omega_{z ; f} l_{z ; f}^{3}=1$. 
and introduce two time dependent parameters $\theta_{b}$ and $\theta_{f}$, which describe the small angular displacements of the bosonic and fermionic cloud, respectively. From the hydrodynamic equations we obtain the equations of motion for the angles

$$
\begin{aligned}
& C_{1} \ddot{\theta}_{b}=D_{11} \theta_{b}+D_{12} \theta_{f} \\
& C_{2} \ddot{\theta}_{f}=D_{21} \theta_{b}+D_{22} \theta_{f} .
\end{aligned}
$$

Although the Eqs.(17) and (18) are formally the same as Eqs. (10) and (11), the coefficients are obviously different. The form of these coefficients is detailed in App A The frequencies of the scissors modes are obtained in a similar way as in Eqs. (12). A typical result is depicted in Fig. 3. Note that similarly to the case of Bose-Bose mixtures, and due to similar reasons, the out-of-phase mode becomes the less energetic state for $0<g_{b f}<g_{b f}^{c}$.

Note that, contrary to the Bose-Bose case, Eq. (16) allows for the possibility of rotational flow, which could be present if the Fermi gas is in the hydrodynamic regime, but still in the normal phase. However, since we are only interested in small deviations with respect to equilibrium, the rotational term, as well as the kinetic energy term, are neglected, since they provide corrections of second order in the angular displacements.
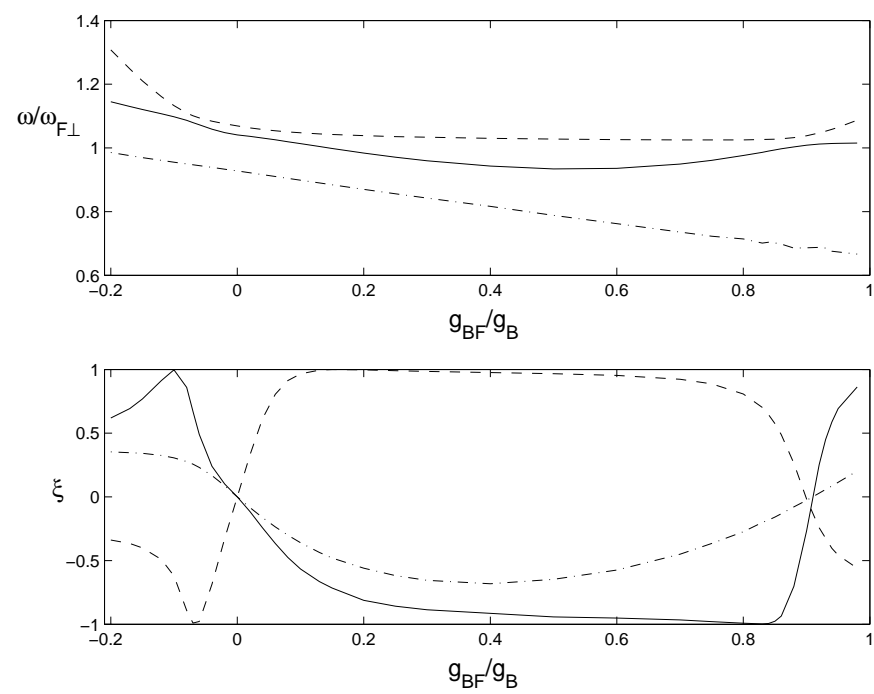

Figure 4: (a) Frequencies of the scissors modes for a FB mixture, where the Fermi gas is in the collisionless regime, , in units of $\omega_{F \perp}=\left(\omega_{x ; f}^{2}+\omega_{y ; f}^{2}\right)^{1 / 2}$, as a function of the intercomponent coupling constant $g_{b f}$; (b) Character of the modes, provided by $\xi=2 \theta_{b} \theta_{f} /\left(\theta_{b}^{2}+\theta_{f}^{2}\right)$, as a function of $g_{b f}$. The inphase (out-of-phase) mode is characterized by $\xi>0(\xi<0)$. Same parameters as in Figure 3 are considered.

\section{B. Collisionless regime}

If the typical time for Fermi-Fermi collisions becomes much smaller that the trapping period, the system enters in the collisionless regime. In this case the bosons still follow the superfluid hydrodynamic equations (13) and (15), but the fermionic gas follows the so-called BoltzmannVlasov equation 31

$$
\frac{\partial f}{\partial t}+\mathbf{v} \cdot \nabla_{\mathbf{r}} f+\frac{1}{m_{f}} \nabla_{\mathbf{r}} U_{f} \cdot \nabla_{\mathbf{v}} f=0,
$$

where $f$ is the phase-space distribution function for the fermions. The external potential, $U_{f}(\mathbf{r})=V_{f}(\mathbf{r})+$ $g_{b f} n_{b}(\mathbf{r})$, contains both the harmonic confinement for the fermions $\left(V_{f}\right)$ and the mean field term due to the interaction with the bosons. The fermionic density is defined as $n_{f}(t, \mathbf{r})=\int d^{3} v f(t, \mathbf{r}, \mathbf{v})$.

Combining the hydrodynamic equations for the bosons and the Boltzmann-Vlasov equation for the fermions, we obtain the following set of equations

$$
\begin{aligned}
& \frac{d}{d t}\langle x y\rangle_{b}=\left\langle x v_{y}+y v_{x}\right\rangle_{b} \\
& \frac{d}{d t}\left\langle x v_{y}+y v_{x}\right\rangle_{b}= \\
& =\left\langle\left[\left(x \partial_{y}+y \partial_{x}\right)\left(\frac{m_{b}}{2} v_{b}^{2}+V_{b}+g_{b} n_{b}+g_{b f} n_{f}\right)\right]\right\rangle_{b} \\
& \frac{d}{d t}\langle x y\rangle_{f}=\left\langle x v_{y}\right\rangle_{f}+\left\langle y v_{x}\right\rangle_{f} \\
& \frac{d}{d t}\left\langle x v_{y}\right\rangle_{f}=\left\langle v_{x} v_{y}\right\rangle_{f}+\frac{1}{m_{f}}\left\langle x\left[\partial_{y}\left(V_{f}+g_{b f} n_{b}\right)\right]\right\rangle_{f} \\
& \frac{d}{d t}\left\langle y v_{x}\right\rangle_{f}=\left\langle v_{x} v_{y}\right\rangle_{f}+\frac{1}{m_{f}}\left\langle y\left[\partial_{x}\left(V_{f}+g_{b f} n_{b}\right)\right]\right\rangle_{f} \\
& \frac{d}{d t}\left\langle v_{x} v_{y}\right\rangle_{f}=\left\langle\left[\left(v_{x} \partial_{y}+v_{y} \partial_{x}\right)\left(V_{f}+g_{b f} n_{b}\right)\right]\right\rangle_{f},
\end{aligned}
$$

where $V_{b}$ is the harmonic confinement for the bosons, $\langle\chi\rangle_{b}=\int \chi(\mathbf{r}) n_{b}(t, \mathbf{r}) d^{3} r / N_{b}$, and $\langle\chi\rangle_{f}=$ $\int \chi(\mathbf{r}, \mathbf{v}) f(t, \mathbf{r}, \mathbf{v}) d^{3} r d^{3} v / N_{f}$.

After a rather tedious algebraic manipulation we arrive to the following equations of motion for the displacement angles (see App. A] :

$$
\begin{aligned}
L_{1} \ddot{\theta}_{b} & =M_{11} \theta_{b}+M_{12} \theta_{f} \\
L_{2} \ddot{\theta}_{f} & =M_{21} \theta_{b}+M_{22} \theta_{f}+M_{23} \eta \\
\ddot{\eta} & =M_{31} \theta_{b}+M_{32} \theta_{f}+M_{33} \eta .
\end{aligned}
$$

Note that in the collisionless regime a third variable couples with the displacement angles, namely $\eta=$ $\int v_{x} v_{y} f d^{3} r d^{3} v / N_{f}$, which is the average value of the deformation in momentum space [27]. Note that in the hydrodynamic regime $\eta=0$, since in the hydrodynamic case the momentum distribution remains always spherically symmetric. A typical case is depicted in Fig. 4 Note the appearance of three scissors modes, and the mixing of the bosonic and the collisional fermionic modes.

\section{CONCLUSIONS}

In this paper we have analyzed the scissors modes obtained after a sudden tilting of the trap axis for the case 
of binary mixtures of quantum gases. We have studied the scissors modes for the case of a BEC with two components, and especially the dependence of the modes with the intercomponent coupling constant. Whereas for attractive intercomponent interactions the in-phase mode always presents the lowest energy, we have shown that for the case of repulsive interactions the character of the modes depends on the density profile of the ground state, and in particular on the overlapping of both components. If both components present the maximal density at the trap center, the lowest energy corresponds to the out-ofphase mode. At a given critical coupling constant one of the components develops a minima at the center, and the in-phase mode becomes the less energetic. A similar reason explains the behavior of other collective excitations in binary mixtures [15].

In the second part of the paper we have focused our attention on the case of a mixture of degenerated bosonic and fermionic gases. We have first analyzed the case in which the fermionic gas is in the hydrodynamic regime, in which the scissors modes present a similar behavior as for the case of binary condensates. We have completed our analysis with the detailed study of the case in which the Fermi gas is in the normal collisionless phase. In that case, the system presents three different scissors modes, due to the asymmetry of the velocity distribution of the fermionic component.

Finally, we would like to stress that the difference between the superfluid hydrodynamic regime and the collisional hydrodynamic regime is provided, from the macroscopic point of view, by the irrotational constraint for the velocity field in the superfluid case $(\nabla \wedge \mathbf{v}=0)$, related to the quantization of the flux and the appearance of quantized vortices. On the contrary, this condition is not necessarily fulfilled in the collisional hydrodynamics. As discussed above, the scissors mode is excited after a sudden change of the trap axis. In the absence of friction such a sudden change cannot induce a rotational flow. Consequently, the scissors modes in collisional hydrodynamics do not differ from those expected in the superfluid case. The differences between collisional and superfluid hydrodynamics in FB mixtures can be revealed extending to the FB mixtures similar ideas as those discussed for Fermi gases in Ref. [32]. This problem will be the subject of further studies.

Note added After the completion of this work, we have learned of a related work performed by Kasamatsu et al. [33] on the problem of scissors modes in binary condensates, in which the authors employ different techniques as those discussed in this paper. In Ref. 33] special emphasis is paid to the nonlinear coupling between quadrupole and scissors modes.

\section{Acknowledgments}

We acknowledge support from Deutsche Forschungsgemeinschaft (SFB 407), the RTN Cold Quantum gases, ESF PESC BEC2000+, and the Ministero dell'Istruzione, dell'Università e della Ricerca (MIUR). M. R. acknowledges the hospitality of the Hannover group. L. S. and P. P. wish to thank the Alexander von Humboldt Foundation, the Federal Ministry of Education and Research and the ZIP Programme of the German Government. M. R. and P. T. thank the Academy of Finland (Project Nos. 53903, 48445) for support.

\section{Appendix A}

The coefficients of Eqs. (10) and (11) can be obtained after a somewhat tedious calculation following the method described in the text, and acquire the form:

$$
\begin{aligned}
B_{1} & =\frac{1}{N_{1}} \int x y n_{1 g}^{\prime} d^{3} r \\
B_{2} & =\frac{1}{N_{2}} \int x y n_{2 g}^{\prime} d^{3} r \\
A_{11} & =\frac{g_{11}}{N_{1}} \int n_{1 g}\left(y \partial_{x}+x \partial_{y}\right) n_{1 g}^{\prime} d^{3} r \\
A_{12} & =\frac{g_{12}}{N_{1}} \int n_{1 g}\left(y \partial_{x}+x \partial_{y}\right) n_{2 g}^{\prime} d^{3} r \\
A_{21} & =\frac{g_{12}}{N_{2}} \int n_{2 g}\left(y \partial_{x}+x \partial_{y}\right) n_{1 g}^{\prime} d^{3} r \\
A_{22} & =\frac{g_{22}}{N_{2}} \int n_{2 g}\left(y \partial_{x}+x \partial_{y}\right) n_{2 g}^{\prime} d^{3} r
\end{aligned}
$$

where $n_{i g}$ denotes the equilibrium density, and $n_{i g}^{\prime}=$ $\left(y \partial_{x}-x \partial_{y}\right) n_{i g}$.

Similarly, we can obtain the coefficients of Eqs. (17) and (18):

$$
\begin{aligned}
C_{1} & =\frac{1}{N_{b}} \int x y n_{1 g}^{\prime} d^{3} r \\
C_{2} & =\frac{1}{N_{f}} \int x y n_{2 g}^{\prime} d^{3} r \\
D_{11} & =\frac{g_{b}}{N_{b}} \int n_{b g}\left(y \partial_{x}+x \partial_{y}\right) n_{b g}^{\prime} d^{3} r \\
D_{12} & =\frac{g_{b f}}{N_{b}} \int n_{b g}\left(y \partial_{x}+x \partial_{y}\right) n_{f g}^{\prime} d^{3} r \\
D_{21} & =\frac{g_{b f}}{N_{f}} \int n_{f g}\left(y \partial_{x}+x \partial_{y}\right) n_{b g}^{\prime} d^{3} r \\
D_{22} & =\frac{g_{f f}}{N_{f}} \int n_{f g}\left(y \partial_{x}+x \partial_{y}\right) \frac{2 n_{f g}^{\prime}}{3 n_{f g}^{1 / 3}} d^{3} r
\end{aligned}
$$

where $g_{f f}=\hbar^{2}(6 \pi)^{2 / 3} / 2 m_{f}$.

Finally, let us briefly discuss the derivation of Eqs. (26), 27) and (28). We assume a velocity field for the fermions of the form

$$
\begin{aligned}
& v_{x}(t, \mathbf{r})=\alpha_{1} y \\
& v_{y}(t, \mathbf{r})=\alpha_{2} x
\end{aligned}
$$


where the time dependence is contained in the parameters $\alpha_{1}$ and $\alpha_{2}$. We additionally define

$$
\begin{aligned}
\left\langle v_{x} v_{y}\right\rangle & =\eta \\
\left\langle x v_{y}+y v_{x}\right\rangle & =\beta .
\end{aligned}
$$

It is possible to rewrite Eqs. (20)-(25) in the form

$$
\begin{aligned}
E_{1} \dot{\theta}_{b} & =\beta \\
\dot{\beta} & =F_{1} \theta_{b}+F_{2} \theta_{f} \\
\left(G_{2}-G_{1}\right) \dot{\theta}_{f} & =G_{1} \alpha_{1}+G_{2} \alpha_{2} \\
G_{1} \dot{\alpha}_{1} & =\eta+Q_{11} \theta_{b}+Q_{12} \theta_{f} \\
G_{2} \dot{\alpha}_{2} & =\eta+Q_{21} \theta_{b}+Q_{22} \theta_{f} \\
\dot{\eta} & =R_{1} \alpha_{1}+R_{2} \alpha_{2}
\end{aligned}
$$

where

$$
\begin{aligned}
E_{1} & =\frac{1}{N_{b}} \int x y n_{b g}^{\prime} d^{3} r \\
F_{1} & =\frac{g_{b}}{N_{b}} \int n_{b g}\left(y \partial_{x}+x \partial_{y}\right) n_{b g}^{\prime} d^{3} r \\
F_{2} & =\frac{g_{b f}}{N_{b}} \int n_{b g}\left(y \partial_{x}+x \partial_{y}\right) n_{f g}^{\prime} d^{3} r \\
G_{1} & =\frac{1}{N_{f}} \int y^{2} n_{f g} d^{3} r \\
G_{2} & =\frac{1}{N_{f}} \int x^{2} n_{f g} d^{3} r \\
Q_{12} & =\int\left[\frac{\omega_{x}^{2}}{N_{f}} x y n_{f g}^{\prime}+\frac{g_{b f}}{N_{f}} n_{f g}^{\prime} y \partial_{x} n_{b g}\right] d^{3} r(
\end{aligned}
$$

$$
\begin{aligned}
Q_{11} & =\frac{g_{b f}}{N_{f}} \int n_{f g} y \partial_{x} n_{b g}^{\prime} d^{3} r \\
Q_{22} & =\int\left[\frac{\omega_{y}^{2}}{N_{f}} x y n_{f g}^{\prime}+\frac{g_{b f}}{N_{f}} n_{f g}^{\prime} x \partial_{y} n_{b g}\right] d^{3} r \\
Q_{21} & =\frac{g_{b f}}{N_{f}} \int n_{f g} x \partial_{y} n_{b g}^{\prime} d^{3} r \\
R_{1} & =\omega_{y}^{2} G_{1}+\frac{g_{b f}}{N_{f}} \int n_{b g} y \partial_{y} n_{b g} d^{3} r \\
R_{2} & =\omega_{x}^{2} G_{2}+\frac{g_{b f}}{N_{f}} \int n_{b g} x \partial_{x} n_{b g} d^{3} r
\end{aligned}
$$

The coefficients of Eqs. (26 28) are of the form

$$
\begin{aligned}
L_{1} & =E_{1} \\
L_{2} & =G_{2}-G_{1} \\
M_{11} & =F_{1} \\
M_{22} & =F_{2} \\
M_{21} & =Q_{11}+Q_{21} \\
M_{22} & =Q_{12}+Q_{22} \\
M_{23} & =2 \\
M_{31} & =\frac{R_{1} Q_{11}}{G_{1}}+\frac{R_{2} Q_{21}}{G_{2}} \\
M_{32} & =\frac{R_{1} Q_{12}}{G_{1}}+\frac{R_{2} Q_{22}}{G_{2}} \\
M_{33} & =\frac{R_{1}}{G_{1}}+\frac{R_{2}}{G_{2}}
\end{aligned}
$$

[1] C. J. Myatt et al, Phys. Rev. Lett. 78, 586 (1997).

[2] J. Stenger et al., Nature 396, 345 (1998).

[3] B. D. Esry, C. H. Greene, J. P. Burke, Jr., and J. L. Bohn, Phys. Rev. Lett. 78, 3594 (1997).

[4] T. Ho, Phys. Rev. Lett. 81, 742 (1998).

[5] P. Öhberg, and S. Stenholm, Phys. Rev. A 57, 1272 (1998).

[6] Th. Busch, J. I. Cirac, V. M. Pérez-García, and P. Zoller, Phys. Rev. A 56, 2978 (1997).

[7] B. D. Esry, and Chris H. Greene, Phys. Rev. A 57, 1265 (1998).

[8] P. Öhberg, Phys. Rev. A 59, 634 (1999).

[9] G. V. Shlyapnikov, Proc. XVIII Int. Conf. on Atomic Physics, Eds.: H. R. Sadeghpour, D. E. Pritchard, and E. J. Heller, (World Scientific Publishing, Singapore, 2002).

[10] A. G. Truscott et al., Science 291, 2570 (2001); F. Schreck et al., Phys. Rev. Lett. 87, 080403 (2001); Z. Hadzibabic et al., Phys. Rev. Lett. 88, 160401 (2002); G. Modugno et al., Science 297, 2240 (2002); Z. Hadzibabic et al., Phys. Rev. Lett. 91, 160401 (2003); K. E. Strecker, G. B. Partridge and R. G. Hulet, Phys. Rev. Lett. 91, 080406 (2003).

[11] K. Mølmer, Phys. Rev. Lett. 80, 1804-1807 (1998).

[12] M. J. Bijlsma, B. A. Heringa and H. T. C. Stoof, Phys. Rev. A 61, 053601 (2000).

[13] H. Pu et al., Phys. Rev. Lett. 88, 070408 (2002).
[14] P. Capuzzi and E. S. Hernández, Phys. Rev. A 64, 043607 (2001).

[15] X.-J. Liu, and H. Hu, Phys. Rev. A 67, 023613 (2003).

[16] A. Albus et al., Phys. Rev. A 65, 053607 (2002).

[17] L. Viverit and S. Giorgini, Phys. Rev. A 66, 063604 (2002).

[18] Giovanni Modugno et al., Science 297, 2240 (2002).

[19] K. K. Das, Phys. Rev. Lett. 90, 170403 (2003); M. A. Cazalilla and A. F. Ho, Phys. Rev. Lett. 91, 150403 (2003).

[20] A. Albus, F. Illuminati and J. Eisert, Phys. Rev. A 68, 023606 (2003); H. P. Büchler and G. Blatter, Phys. Rev. Lett. 91, 130404 (2003); A. B. Kuklov and B. V. Svistunov, Phys. Rev. Lett. 90, 100401 (2003); M. Lewenstein, L. Santos, M. Baranov and H. Fehrmann, cond-mat/0306180 H. Fehrmann et al., cond-mat/0307635 R. Roth and K. Burnett, cond-mat/0310114

[21] D. S. Jin et al., Phys. Rev. Lett. 77, 420 (1996).

[22] M.-O. Mewes, M.R. Andrews, N.J. van Druten, D.M. Kurn, D.S. Durfee, C.G. Townsend, and W. Ketterle, Phys. Rev. Lett. 77, 988 (1996).

[23] K.G. Singh and D.S. Rokhsar, Phys. Rev. Lett. 77, 1667 (1996); M. Edwards et al., Phys. Rev. Lett. 77, 1671 (1996); S.Stringari, Phys. Rev. Lett. 77, 2360 (1996); L. You, W. Hoston, and M. Lewenstein, Phys. Rev. A 55, 
R1581 (1997); P. Öhberg et al. , Phys. Rev. A 56, R3346 (1997).

[24] V. M. Pérez-García et al., Phys. Rev. Lett. 77, 5320 (1996).

[25] T. Bergeman, Phys. Rev. A 55, 3658 (1997).

[26] N. Lo Iudice and F. Palumbo, Phys. Rev. Lett. 41, 1532 (1978); E. Lipparini and S. Stringari, Phys. Lett. 130B, 139 (1983).

[27] D. Guéry-Odelin and S. Stringari, Phys. Rev. Lett. 83, 4452 (1999).

[28] O. M. Maragò et al, Phys. Rev. Lett. 84, 2056 (2000).
[29] M. Modugno et al., Phys. Rev. A 67, 023608 (2003).

[30] L. Vichi and S. Stringari, J. Low. Temp. Phys. 121, 177 (2000).

[31] K. Huang, Statistical Mechanics, 2nd ed. (Wiley, New York, 1987).

[32] M. Cozzini and S. Stringari Phys. Rev. Lett. 91, 070401 (2003).

[33] K. Kasamatsu, M. Tsubota, and M. Ueda, cond-mat/0309539 